\title{
If it looks, sounds, or feels like subitizing, is it subitizing? A modulated definition of subitizing
}

\author{
Naama Katzin ${ }^{1,2} \cdot$ Zahira Ziva Cohen $^{1} \cdot$ Avishai Henik ${ }^{1,2}$ \\ Published online: 10 January 2019 \\ (C) The Psychonomic Society, Inc. 2019
}

\begin{abstract}
Research in cognitive psychology has focused mainly on the visual modality as the input interface for mental processes. We suggest that integrating studies from different modalities can aid in resolving theoretical controversies. We demonstrate this in the case of subitizing. Subitizing, the quick and accurate enumeration of small quantities, has been studied since the 19th century. Nevertheless, to date, the underlying mechanism is still debated. Two mechanisms have been suggested: a domain-general mechanism - attention, and a domain-specific mechanism - pattern recognition. Here, we review pivotal studies in the visual, tactile, and auditory modalities. The accumulative findings shed light on the theoretical debate. Accordingly, we suggest that subitizing is a subprocess of counting that occurs in the presence of facilitating factors, such as attentional resources and familiar patterns.
\end{abstract}

Keywords Subitizing $\cdot$ Pattern recognition $\cdot$ FINST theory $\cdot$ Multimodal

Theoretical frameworks in cognitive psychology mostly rely on the visual modality as the window to human cognition. The widespread use of the visual modality is quite natural and logical, as vision is humans' most dominant path to experience the outside world and to internally represent it. Accordingly, a large portion of the brain is dedicated to vision and visual-related processes (e.g., Atkinson, 2002). Yet it is hard to disagree that we experience the world with all our senses; when we stroll in the woods, we can smell the fresh scent of the wet soil, we hear the sounds of the crackling leaves beneath our shoes, and we feel the cold breeze on our body. All these sensory cues lead us to the notion that winter is coming. General cognitive processes are considered to be either specific to the visual modality, or are assumed to be amodal, although the latter assumption is not always validated.

Naama Katzin and Zahira Ziva Cohen contributed equally to this work and order was assigned randomly .

Zahira Ziva Cohen zahiraci@gmail.com

1 Department of Psychology, Ben-Gurion University of the Negev, P.O.B: 953, Beer-Sheva, Israel

2 Zlotowski Center for Neuroscience, Ben-Gurion University of the Negev, Beer-Sheva, Israel
In this paper, we use subitizing - fast and accurate enumeration of small quantities, as a prime example for the added value of integrating findings from different modalities.

In 1859, Hamilton posed the philosophical question, "How many objects can the mind embrace at once?" (p. 176). He hypothesized it would be six objects, but he did not put it to test. Twelve years later, Jevons (1871) addressed this issue experimentally. He repeatedly threw a handful of black beans on a tray and tried to estimate their quantity. After each throw, he recorded the actual number of beans on the tray and his quick estimation. His results showed that four objects are perceived quickly and accurately. Two centuries later this question is still very much alive. Inspired by Jevons, most of the efforts made to address this question focused, naturally, on the visual domain. The sparse studies in other modalities (tactile and auditory) searched for the pattern that was found in the visual modality, without taking into consideration basic differences between modalities, resulting in inconsistency in findings.

In what follows, we review the pivotal studies in each modality (visual, tactile and auditory; see Fig. 1). We will elaborate on two main hypothesized theoretical mechanisms of subitizing, and their manifestations in other modalities. We will focus on (1) the varying range of subitizing within and between modalities; (2) the methodology used in each modality; and (3) the relations between subitizing and counting as 


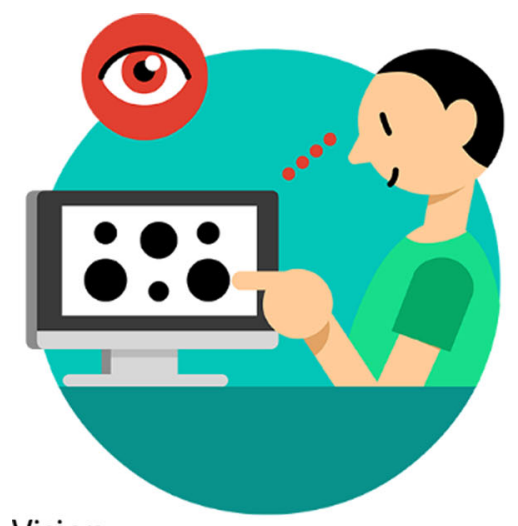

Vision:

- Presentation: Simultaneous

- Subitizing range: 4

- Higher range for canonical patterns

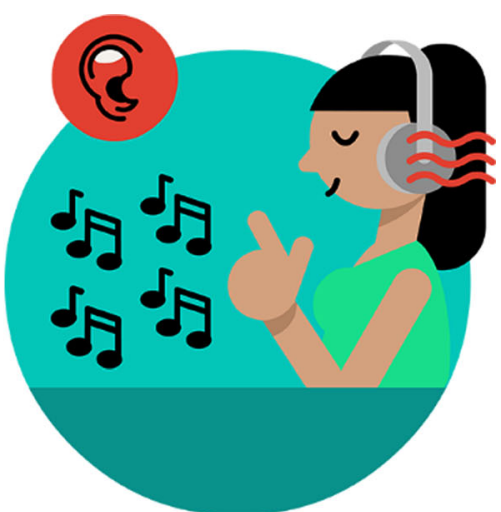

Audition:

- Presentation: Sequential

- Subitizing range: 2-3

- Familiar temporal patterns modulate enumeration

Tactile:

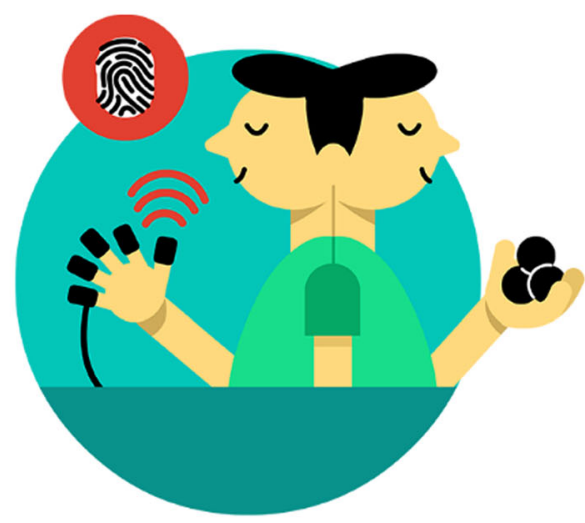

- Presentation: Simultaneous

- Subitizing range: 3 to 6

- Quick and accurate enumeration of finger counting patterns such as 5 and 10

Fig. 1 Summary of the literature on subitizing in three modalities. Vision: Participants are instructed to enumerate dots that appear on a screen. Audition: Participants are instructed to enumerate the number of sounds they hear. Tactile: In the passive paradigm, participants are required to

guiding cues to our understanding of the subitizing phenomenon, and consequentially, to our suggestion of an a-modal, parsimonious modulated definition of subitizing.

\section{Subitizing in the visual domain}

In the 19th century, Hamilton and Jevons toyed with the question regarding the number of objects that can be perceived simultaneously. With the development of perception and attention theories and the establishment of psychology as a scientific field, the question was re-examined. Saltzman and Garner (1948) sought to find the span of attention. They concluded that the span of attention is not a constant but a variable. In a series of experiments, they showed that exposure time, prior knowledge and practice modulated the span. Kaufman and enumerate the number of fingers that are stimulated. In the active paradigm, participants are asked to feel and enumerate the number of objects in their hand.

colleagues coined the term subitizing to describe fast, accurate, and confident enumeration of quantities below six (Kaufman, Lord, Reese, \& Volkmann, 1949). When numerosities of six and above are enumerated, the process is more time consuming, less accurate, and is carried out with less confidence. Depending on task demands, objects are either enumerated in a serial slow and accurate process (i.e., counting), or enumerated quicker with more errors (i.e., estimation). Fast response times (RT) and high accuracy rates became the operational definition of subitizing and the standard criteria.

From this definition, four methods for calculating the subitizing range were derived. The first method is rather straightforward: exploratory RT slope analysis. RT slopes are calculated between every two subsequent numerosities; $\mathrm{RT}_{(\mathrm{n}+}$ ${ }_{11}-\mathrm{RT}_{(\mathrm{n})}$. The slopes are compared with an analysis of variance. The subitizing range is defined where there is a significant 
difference between the two slopes (see Fig. 2a). The second method is fitting a bilinear function. In this method, subitizing and counting are characterized by two distinct linear functions. The subitizing linear function has a significantly smaller RT slope than the RT slope of the counting range. The point at which these two functions split is the marker of the subitizing range (see Fig. 2b; e.g., Green \& Bavelier, 2003). The third method is fitting a sigmoid function. According to this method, RT enumeration data is best characterized with a sigmoid curve, in which the inflection point marks the subitizing range (see Fig. 2c; e.g., Piazza, Fumarola, Chinello, \& Melcher, 2011). Lastly, the fourth method, bilinear sigmoid fit, incorporates both the bilinear function and the sigmoid function (Leibovich-Raveh, Lewis, Al-Rubaiey Kadhim, \& Ansari, 2018). Importantly, these methods were intended to find a single breaking point between subitizing and counting, assuming that the subitizing range precedes the counting range and that subitizing is characterized by numerical contiguity.

The exact range of subitizing was debated for some time (e.g., Basak \& Verhaeghen, 2003; Kaufman et al., 1949; Landerl, Bevan, \& Butterworth, 2004; Mandler \& Shebo, 1982; Piazza, Mechelli, Butterworth, \& Price, 2002; Saltzman \& Garner, 1948). Nowadays, visual subitizing refers to the perception of quantities between one and four.

After the characteristics of subitizing were agreed upon, one question that remained open was what is the underlying mechanism of subitizing? There are two competing notions; domain-general and domain-specific. One main domaingeneral theory suggests that limitation of attention could account for the four-item limit of subitizing. In 1994, Trick and Pylyshyn proposed the Fingers of INSTantiation (FINST) theory, according to which subitizing is a product of a limitedcapacity parallel mechanism of item individuation (Trick \& Pylyshyn, 1994). They postulated that there are around four mental indexes that can pick out and stay attached to objects when searching the visual field, regardless of their changing properties. According to the FINST theory, subitizing was considered to be automatic and pre-attentional, though numerous studies showed that subitizing does require attention (e.g., in Anobile, Turi, Cicchini, \& Burr, 2012; Burr, Turi, \& Anobile, 2010; Egeth, Leonard, \& Palomares, 2008; Gliksman, Weinbach, \& Henik, 2016; Olivers \& Watson, 2008; Pagano, Lombardi, \& Mazza, 2014; Railo, Koivisto, Revonsuo, \& Hannula, 2008) and is dependent on the stimuli properties (Saltzman \& Garner, 1948).

An important domain-specific theory, pattern recognition, suggests that spatial organization of objects enhances enumeration of small quantities. In 1982, Mandler and Shebo suggested that quantities of up to four stimuli create familiar patterns: two dots create a line, three dots create a triangle, and four dots create a quadrilateral (Mandler \& Shebo, 1982). Accordingly, numerosities that were presented in canonical arrangements (familiar patterns as in a dice; see Fig. 3) were found to be enumerated more efficiently even above the defined subitizing range (Ashkenazi, Mark-Zigdon, \& Henik, 2013; Piazza et al., 2002). Moreover, familiarity, which was manipulated using a training procedure, showed that even 18 randomly scattered dots, which were repeatedly enumerated during training, were enumerated fast and accurately (Wolters, van Kempen, \& Wijlhuizen, 1987).

These theories were examined and discussed not just using behavioral methods but also with neuroimaging methods (e.g., in Cutini, Scatturin, Basso Moro, \& Zorzi, 2014; Leibovich, Henik, \& Salti, 2015; Piazza et al., 2002), with abnormal populations (Ashkenazi et al., 2013; Bagattini et al., 2017; Dehaene \& Cohen, 1994; Gagnon, Mottron, Bherer, \& Joanette, 2004; Mazza, 2017), and in other modalities.

Nonetheless, the debate between the two theories has not been resolved yet. Intriguingly, neither theory can solely account for one observation: RT pattern in the subitizing range has a slope, a very small slope, and significantly smaller than the counting RT slope, but distinct. In other words, one item is enumerated faster than two, and so on. According to FINST theory, subitizing is a parallel preattentive process. Accordingly, up to four stimuli, RTs should not be affected. Poiese, Spalek, and Di Lollo (2008) hypothesized that if subitizing is preattentive and parallel, then it should (a) occur
(A)

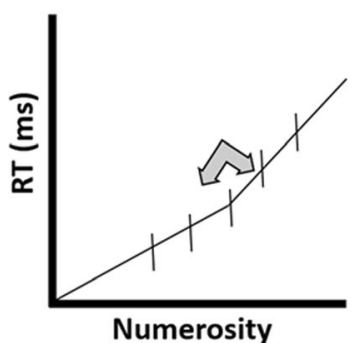

(B)

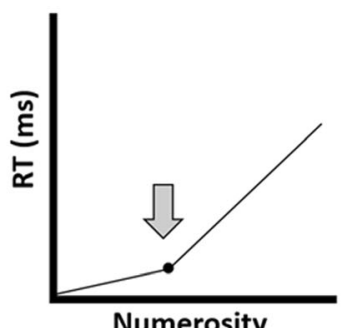

Numerosity
(C)

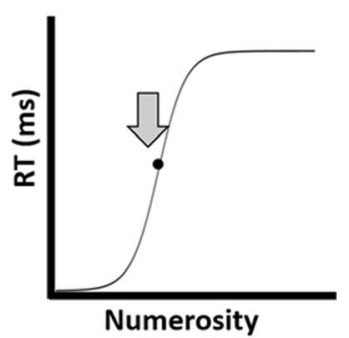

Fig. 2 Example of three methods for calculating the subitizing range. a Exploratory RT slope analysis; the arrows point to the slopes that are compared. b Bilinear function; the black dot marks the upper boundary of the subitizing range, where the two linear functions split. c Sigmoid function; the black dot marks the upper boundary of the subitizing range at the inflection point. 


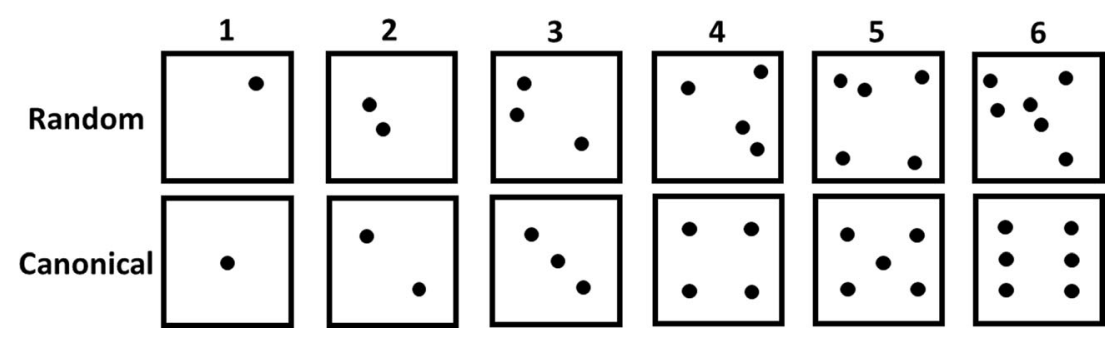

Fig. 3 Examples of random and canonical arrangements of numerosities 1-6

within $100 \mathrm{~ms}$ of the presentation of the display, and (b) performance should be similar for the entire subitizing range. To test their hypothesis, they presented participants with displays to be enumerated and manipulated the stimulus onset asynchrony (SOA) between the display and the mask. Their results showed that longer SOAs generated higher accuracy rates and sensitivity scores ( $d$ '). Also, for one stimulus display, the performance was better than a display of two stimuli. Alternately, according to the pattern recognition theory, small quantities are "chunked" into a single pattern, and therefore are enumerated in one "mental beat." If the process of subitizing is indeed a distinct process, based on "at once" perception per se, then it should not be affected by specific patterns and there should not be any RT slope.

We will now review the findings from other modalities, and try to see if they can enrich the theoretical debate, and assist in resolving the discrepancies.

\section{Subitizing in the tactile domain}

The input surface of the tactile modality is the skin. It is known that tactile sensitivity varies for different body parts, as demonstrated by the somatosensory homunculus (Maeno, Kobayashi, \& YamaZaki, 1997; Nakamura et al., 1998; Narici et al., 1991; Yang, Gallen, Schwartz, \& Bloom, 1993). Accordingly, the enumeration process manifestation depends on the body part used. Moreover, there are different types of tactile inputs (pressure, vibration, heat, etc.) that are processed through distinct receptors (Caldwell, Tsagarakis, \& Wardle, 1997; Chouvardas, Miliou, \& Hatalis, 2005; Chouvardas, Miliou, \& Hatalis, 2008; Gallace \& Spence, 2014). Furthermore, specific body parts (i.e., the fingers) have numerical associations - an embodiment (Fischer \& Brugger, 2011). Embodiment (i.e., embodied cognition, or specifically, embodied numerosity) refers to abstract cognitive representations associated with senso-motoric experiences (Barsalou, 2008; Wilson, 2002); the use of finger counting during childhood shapes the way we process numbers abstractly. For example, the use of fingers has been suggested to be the origin of the base-10 Hindu-Arabic numeral system (Di Luca \& Pesenti, 2011). The variability in sensitivity, input surface and embodied characteristics is mirrored in the literature, resulting in inconsistent findings and conclusions.

The first to search for subitizing in the tactile modality were Riggs et al. (2006). They used pressure stimuli that were applied simultaneously to the fingertips of both hands and concluded that the tactile subitizing range is 3 . They suggested that FINST theory can account for the results. Plaisier and Smeets (2011) showed that subitizing of three stimuli manifests when the to-be-enumerated fingers are the only fingers that receive tactile information. When using vibrations to the fingertips, Cohen and Henik (2016) found subitizing of 2 stimuli; counting from 3 to 6 ; accelerated counting from 7 to 9 ; and quick and accurate (i.e., efficient) responses for 10 stimuli. Other than the type of stimuli, more differences could account for the variability in results. For example, Plaisier and Smeets excluded the thumbs and Cohen and Henik used all 10 fingers, activating fingernumber associations (Cohen \& Henik, 2016; Plaisier \& Smeets, 2011). This activation was also demonstrated in one hand, with subjects showing an efficient response to five stimuli. Also, applying stimuli to neighboring fingers yielded more efficient responses (Cohen, Naparstek, \& Henik, 2014). Cohen and colleagues suggested that these results support the pattern recognition theory, since the familiar finger counting patterns showed increased efficiency (Cohen et al., 2018; Cohen, Aisenberg, \& Henik, 2016; Cohen \& Henik, 2016; Cohen et al., 2014)

Active touch studies (i.e., enumeration with active fingers exploration of objects) using one hand (Plaisier, Tiest, \& Kappers, 2009) found subitizing of three objects. Active touch using two hands showed that subitizing of 3 could co-occur in both hands, perceptually "chunked," generating a total subitizing range of 6 (Plaisier, Tiest, \& Kappers, 2010). Only one study examined enumeration using other body parts and reported an absence of subitizing (Gallace, Tan, \& Spence, 2008).

\section{Subitizing in the auditory domain}

Three studies explicitly examined subitizing in the auditory modality (Camos \& Tillmann, 2008; Repp, 2007; ten Hoopen \& Vos, 1979). All these studies presented sounds sequentially 
and not simultaneously. Subitizing can also be considered a sequential process in which sequentially presented items are labeled through the use of a related process called rhythm (i.e., sequential subitizing; Davis \& Pérusse, 1988, p. 562). The justifications for the sequential presentation is that simultaneous presentation creates acoustic confounds (Camos \& Tillmann, 2008), and more convincing, stimuli in the auditory modality in real life are usually, sequential (Repp, 2007; ten Hoopen \& Vos, 1979). Yet sequential presentation encourages a counting strategy. In order to avoid this, stimuli are presented in a fast sequence that prevents counting.

The first to examine auditory subitizing were ten Hoopen and Vos (1979). They introduced a series of tones chunked into subgroups (2-7 tones). In half of the trials, there was a change in frequency between the subgroups (i.e., grouped series), and in the other half there was no change (i.e., ungrouped series). Subjects were instructed to report the number of sounds in a series. The underlying assumption was that to report the number of sounds in a series, subjects assessed the number of sounds in a subgroup and multiplied it by the number of subgroups. This process is considered to be simpler in grouped series, in which the subgroups are easily distinguished because of the change in frequency. Accordingly, the authors argued that in grouped series, errors should be attributed to a failure in estimating how many sounds were in a subgroup, and not to counting the number of subgroups. Indeed, accuracy rates for 2-3 tones were higher for grouped series (vs. ungrouped series). The authors concluded that auditory subitizing is 3 . It is important to note that the accuracy of 2-3 tones was only $20 \%$.

The study of Repp (2007) was the first to measure both accuracy and response time (RT). He examined auditory subitizing in musicians and non-musicians. For musicians, accuracy deteriorated at six sounds, and RTs did not "show the telltale signs of subitizing" (p. 538). For non-musicians, he found high accuracy and fast RTs for 2-3 tones. He suggested that the difference in accuracy between musicians and nonmusicians highlights that subitizing is an ability that is shaped by experience. He proposed that a possible underlying mechanism is temporal pattern recognition, which stems from the commonly used time signatures (i.e., meters - rhythmic structure) in Western music that are made up of pairs and triplets of sounds (e.g., $3 / 4$ is $2+2+2,6 / 8$ is $3+3$ ).

Camos and Tillman (2008) compared auditory subitizing to visual subitizing; both presented sequentially. They found a subitizing range of 2 in both modalities. Interestingly, they showed greater accuracy and faster responses in audition. They interpreted this similarity between visual and auditory subitizing as evidence for a general attention capacity limitation.

To the best of our knowledge, these are the only studies on subitizing in the auditory modality. None of them used simultaneous presentation, which is a main feature in the formal definition of subitizing (Kaufman et al., 1949). However, even Jevons, in the classic paper from 1871, concluded that the notion of limited capacity of numerical discrimination is supported by the principles of rhythm ("recognizable rhythmic pattern" are also discussed in Davis \& Pérusse, 1988; von Glasersfeld, 1982). Nevertheless, with regards to simultaneous presentation, one possible experiment could compare enumeration of harmonic and disharmonic patterns of notes. If indeed subitizing relies on recognition of familiar patterns, the subitizing range might vary between harmonic and disharmonic patterns, and between musicians and non-musicians.

\section{Discussion}

Reviewing the literature of subitizing in the three modalities reveals inconsistencies regarding the subitizing range, the methods used, and the assumed underlying mechanism. There is no substantial agreement on the subitizing range within the tactile and auditory modalities, let alone between the three modalities; in the visual modality, the subitizing range is defined as 4 , but presenting familiar patterns can increase the range. In the tactile modality, the range is dependent on various aspects such as input surface (fingers or other body parts), stimulus type (pressure or vibration), and utilization (one hand, both hands, passive or active touch). On the fingers, where it is studied most, the range is either 3 or 4 , but fast and accurate responses were found for 5, 6, and 10 stimuli as well. In the auditory modality, the sounds to enumerate were presented sequentially and the subitizing range is 2,3 , or 6 for musicians. In addition, expanding the research to modalities other than the visual did not reconcile the debate between the two competing theories: domain-general or domain-specific mechanisms. In what follows, we suggest a synergized framework of subitizing that is based on integration of findings from all modalities.

As mentioned earlier, there are two prominent theories regarding subitizing: one is domain-general (FINST; attention) and the other is domain-specific (pattern recognition). Even though these theories are based on visual findings, they are not considered exclusive to visual subitizing and were used, alternately and inconsistently, to explain the results in other modalities. But can each theory, by itself, account for the variability in the subitizing range within and between modalities?

With regards to the FINST theory, one hypothesis might be that if indeed subitizing is limited by a domain-general factor, the input channel should not matter. In other words, if the subitizing limit is due to general attentional constrains, the range should be the same for all modalities (Camos \& Tillmann, 2008). The results suggest differently, as the subitizing range is not the same for all modalities. Nevertheless, we are not ready to dismiss the domaingeneral theory. While attention is a general resource, it is 
still limited by the input channel. The processing speed and body-surface-brain pathways of different modalities is markedly different (Møller, 2003). Although the role of a domain-general factor cannot be ruled out, the FINST theory seems insufficient. FINST theory limits subitizing to 4 , but we do find fast and accurate enumeration of numerosities larger than 4.

What characterizes the fast and accurate large subitized quantities? The common characteristic of these "subitized" large numerosities is that they create recognizable patterns. If the basic mechanism of subitizing is pattern recognition, then any quantity that constitutes a recognizable pattern can be subitized. For example, enumerating five tactilely presented stimuli using one hand or 10 using both hands is a recognized embodied finger-counting pattern. Moreover, we are more efficient in enumerating neighboring arrangements, as it is the way we usually count with our fingers (Cohen, Aisenberg, \& Henik, 2016; Cohen \& Henik, 2016; Cohen et al., 2014; Rinaldi, Di Luca, Henik, \& Girelli, 2016). Visual canonical arrangements facilitate enumeration since they are strongly associated with a quantity (Mandler \& Shebo, 1982), and as was suggested by Repp (2007), commonly used rhythms may constitute recognized temporal patterns that affect auditory subitizing. These unexpected subitized quantities support the domain-specific mechanism of pattern recognition.

The old notion of "chunking" (Miller, 1956) may aid in synthesizing the attentional limit theory with pattern recognition theory. Miller once referred to the number 7-the capacity of working memory, as if he was "persecuted by an integer" (p. 81). He suggested that the capacity of information processing in immediate memory is affected by the ability to group inputs into familiar units, or "chunks." Inspired by Miller, we suggest that the span of attention is limited to a specific range, but an item can be more than a single stimulus due to learning or familiarity; as high as 18 random dots that were trained (Wolters et al., 1987) and 6 (3 in each hand) spheres were perceptually grouped (Overvliet \& Plaisier, 2016). Thus, we suggest that, although limited, there is no specific integer to represent attention capacity. For our purposes, a recognized pattern can be considered as a single complex item in terms of attention capacity and individuation, and could be subitized. Therefore, the question of "how many objects can be perceived at once?" cannot be answered with a specific number.

The confusion with regards to the different ranges within and between modalities is embedded in the classic definition of subitizing, which was, and still is, visually biased; restricting the subitizing range to small quantities. Imposing this definition on other modalities (e.g., by expecting the same range, accuracy rates, RT slope, and the same RT pattern with one "break" between subitizing RT and counting RT) is like fitting a square peg in a round hole. The importance of unfolding the variability in the reviewed literature, holding in mind the philosophical question, urges us to take a step back and create a unified, more parsimonious a-modal definition of subitizing.

In our attempt to do so, we would like to point out another controversy in the field of enumeration: Are subitizing and counting two distinct phenomena, or are they a manifestation of a single process that varies in efficiency (e.g., Balakrishnanl \& Ashby, 1992; Gallace \& Spence, 2008, 2014; Piazza et al., 2002; Sengupta, Bapiraju, \& Melcher, 2016)? In light of our review, showing that large quantities in different modalities are also subitized, it seems reasonable to collapse the two processes to a unified and continuous process that can be accelerated when facilitating factors exist. We suggest that even in the subitizing range there is a counting process, but it is facilitated for various reasons: available attentional resources, a familiar pattern, and so on. This may account for the slope within the subitizing range. To illustrate, imagine that you pay a daily visit to your local ice-cream shop. Every day you order your favorite flavor, salted caramel. Once the salesman learns that this is your usual order, when he sees you enter the store he prepares (mentally andlor physically) to serve this flavor. Accordingly, the service improves due to a facilitating factor, familiarity.

One does not have to agree with our interpretation of the results. Still, this review can teach us about the advantages of studying the same phenomenon in different modalities. Multimodal studies can determine how general (i.e., a-modal) a certain cognitive mechanism is. Moreover, the findings from several modalities can enrich theoretical debates. However, when adapting an experimental design created for a specific modality to another modality, one needs to take into consideration modality dependent factors, holding in mind the question that needs to be answered. These two considerations do not always coincide. For example, enforcing visual characteristics of RT on the tactile modality, does not allow 5 or 10 to be considered subitized, although they are enumerated fast and accurately.

To conclude, in the case of subitizing, incorporating findings across modalities corroborates the idea of a single enumeration mechanism. According to this approach, we suggest a modified and parsimonious definition of subitizing; a subprocess of counting that takes place when facilitating factors are present, and yields accelerated and more accurate enumeration. This definition can account for variability both within and between modalities and can answer the question of "how many" with the most anoying, yet true, answer: "It depends."

Acknowledgements This work was supported by the European Research Council under the European Union's Seventh Framework Programme (FP7/2007-2013)/ERC Grant Agreement No. 295664 awarded to Avishai Henik, and by the Israel Science Foundation (Grant 1799/12) in the framework of their Centers of Excellence. Special thanks are given to Desiree Meloul for her professional and generous help. 
Publisher's Note Springer Nature remains neutral with regard to jurisdictional claims in published maps and institutional affiliations.

\section{References}

Anobile, G., Turi, M., Cicchini, G. M., \& Burr, D. C. (2012). The effects of cross-sensory attentional demand on subitizing and on mapping number onto space. Vision Research, 74, 102-109. doi:https://doi. org/10.1016/j.visres.2012.06.005

Ashkenazi, S., Mark-Zigdon, N., \& Henik, A. (2013). Do subitizing deficits in developmental dyscalculia involve pattern recognition weakness? Developmental Science, 16(1), 35-46. doi:https://doi. org/10.1111/j.1467-7687.2012.01190.x

Atkinson, J. (2002). The developing visual brain. Oxford: Oxford University Press. Retrieved from https://philpapers.org/rec/ ATKTDV

Bagattini, C., Mazza, V., Panizza, L., Ferrari, C., Bonomini, C., \& Brignani, D. (2017). Neural dynamics of multiple object processing in mild cognitive impairment and Alzheimer's disease: Future early diagnostic biomarkers? Journal of Alzheimer's Disease, 59(2), 643654. doi:https://doi.org/10.3233/JAD-161274

Balakrishnanl, J. D., \& Ashby, F. G. (1992). Subitizing: Magical numbers or mere superstition? Psychological Research, 54(2), 80-90. doi: https://doi.org/10.1007/BF00937136

Barsalou, L. W. (2008). Grounded cognition. Annual Review of Psychology, 59(1), 617-645. doi:https://doi.org/10.1146/annurev. psych.59.103006.093639

Basak, C., \& Verhaeghen, P. (2003). Subitizing speed, subitizing range, counting speed, the Stroop effect, and aging: Capacity differences and speed equivalence. Psychology and Aging, 18(2), 240-249. doi: https://doi.org/10.1037/0882-7974.18.2.240

Burr, D. C., Turi, M., \& Anobile, G. (2010). Subitizing but not estimation of numerosity requires attentional resources. Journal of Vision, 10(6), 20. doi:https://doi.org/10.1167/10.6.20

Caldwell, D., Tsagarakis, N., \& Wardle, A. (1997). Mechano thermo and proprioceptor feedback for integrated haptic feedback. In Proceedings of International Conference on Robotics and Automation (pp. 2491-2496). doi:10.1109/ROBOT.1997.619335

Camos, V., \& Tillmann, B. (2008). Discontinuity in the enumeration of sequentially presented auditory and visual stimuli. Cognition, 107(3), 1135-1143. doi:https://doi.org/10.1016/j.cognition.2007.11.002

Chouvardas, V., Miliou, A., \& Hatalis, M. K. (2005). Tactile displays: A short overview and recent developments. In ICTA '05: Proceedings of Fifth International Conference on Technology and Automation (pp. 246-251).

Chouvardas, V. G., Miliou, A. N., \& Hatalis, M. K. (2008). Tactile displays: Overview and recent advances. Displays, 29(3), 185-194. doi:https://doi.org/10.1016/j.displa.2007.07.003

Cohen, Z. Z., Aisenberg, D., \& Henik, A. (2016). The effects of training on tactile enumeration. Psychological Research, 1-20. Advance online publication. doi:https://doi.org/10.1007/s00426-016-0835-5

Cohen, Z., Arend, I., Yuen, K., Naparstek, S., Gliksman, Y., Veksler, R., \& Henik, A. (2018). Tactile enumeration: A case study of acalculia. Brain and Cognition, 127(7), 60-71. doi:https://doi.org/10.1016/j. bandc.2018.10.001

Cohen, Z. Z., \& Henik, A. (2016). Effects of numerosity range on tactile and visual enumeration. Perception, 45(1-2), 83-98. doi:https://doi. org/10.1177/0301006615614662

Cohen, Z. Z., Naparstek, S., \& Henik, A. (2014). Tactile enumeration of small quantities using one hand. Acta Psychologica, 150, 26-34. doi:https://doi.org/10.1016/j.actpsy.2014.03.011

Cutini, S., Scatturin, P., Basso Moro, S., \& Zorzi, M. (2014). Are the neural correlates of subitizing and estimation dissociable? An
fNIRS investigation. NeuroImage, 85, 391-399. doi:https://doi. org/10.1016/j.neuroimage.2013.08.027

Davis, H., \& Pérusse, R. (1988). Numerical competence in animals: Definitional issues, current evidence, and a new research agenda, Behavioral and Brain Sciences, 11(4), 561-579.

Dehaene, S., \& Cohen, L. (1994). Dissociable mechanisms of subitizing and counting: Neuropsychological evidence from simultanagnosic patients. Journal of Experimental Psychology: Human Perception and Performance, 20(5), 958-975. doi:https://doi.org/10.1037/ 0096-1523.20.5.958

Di Luca, S., \& Pesenti, M. (2011). Finger numeral representations: More than just another symbolic code. Frontiers in Psychology. doi: https://doi.org/10.3389/fpsyg.2011.00272

Egeth, H. E., Leonard, C. J., \& Palomares, M. (2008). The role of attention in subitizing: Is the magical number 1? Visual Cognition, 16(4), 463-473. doi:https://doi.org/10.1080/13506280801937939

Fischer, M. H., \& Brugger, P. (2011). When digits help digits: Spatialnumerical associations point to finger counting as prime example of embodied cognition. Frontiers in Psychology, 2(October), 41-47. doi:https://doi.org/10.3389/fpsyg.2011.00260

Gagnon, L., Mottron, L., Bherer, L., \& Joanette, Y. (2004). Quantification judgement in high functioning autism: Superior or different? Journal of Autism and Developmental Disorders, 34(6), 679-689. doi:https://doi.org/10.1007/s10803-004-5288-9

Gallace, A., \& Spence, C. (2008). The cognitive and neural correlates of "tactile consciousness": A multisensory perspective. Consciousness and Cognition, 17(1), 370-407. doi:https://doi.org/10.1016/j. concog.2007.01.005

Gallace, A., \& Spence, C. (2014). In touch with the future: The sense of touch from cognitive neuroscience to virtual reality. Oxford: Oxford University Press. doi:https://doi.org/10.1111/bjop.12088

Gallace, A., Tan, H., \& Spence, C. (2008). Can tactile stimuli be subitised? An unresolved controversy within the literature on numerosity judgments. Perception, 37(5), 782-800. doi:https://doi. org/10.1068/p5767

Gliksman, Y., Weinbach, N., \& Henik, A. (2016). Alerting cues enhance the subitizing process. Acta Psychologica, 170, 139-145. doi: https://doi.org/10.1016/j.actpsy.2016.06.013

Green, C. S., \& Bavelier, D. (2003). Action video game modifies visual selective attention. Nature, 423(May), 534-537. doi:https://doi.org/ 10.1038 /nature 01647

Hamilton, W. (1859). Lectures on metaphysics and logic (Vol. 1). Washington, DC: Gould and Lincoln.

Jevons, S. W. (1871). The power of numerical discrimination. Nature, 3, 281-282. doi:https://doi.org/10.1038/003281a0

Kaufman, E. L., Lord, M. W., Reese, T. W., \& Volkmann, J. (1949). The discrimination of visual number. The American Journal of Psychology, 62(4), 498-525. doi:https://doi.org/10.2307/1418556

Landerl, K., Bevan, A., \& Butterworth, B. (2004). Developmental dyscalculia and basic numerical capacities: A study of 8-9-yearold students. Cognition, 93(2), 99-125. doi:https://doi.org/10. 1016/j.cognition.2003.11.004

Leibovich, T., Henik, A., \& Salti, M. (2015). Numerosity processing is context driven even in the subitizing range: An fMRI study. Neuropsychologia, 77, 137-147. doi:https://doi.org/10.1016/j. neuropsychologia.2015.08.016

Leibovich-Raveh, T., Lewis, D. J., Al-Rubaiey Kadhim, S., \& Ansari, D. (2018). A new method for calculating individual subitizing ranges. Journal of Numerical Cognition, 4(2), 429-447. doi:https://doi.org/ 10.5964/jnc.v4i2.74

Maeno, T., Kobayashi, K., \& YamaZaki, N. (1997). Relationship between structure of finger tissue and location of tactile receptors. Nippon Kikai Gakkai Ronbunshu, C Hen/Transactions of the Japan Society of Mechanical Engineers Part C, 63(607), 881-888. 
Mandler, G., \& Shebo, B. J. (1982). Subitizing: An analysis of its component processes. Journal of Experimental Psychology: General, 111(1), 1-22. doi:https://doi.org/10.1037/0096-3445.111.1.1

Mazza, V. (2017). Simultanagnosia and object individuation. Cognitive Neuropsychology, 1-10. Advance online publication. doi:10.1080/ 02643294.2017 .1331212

Miller, G. A. (1956). The magical number seven, plus or minus two: Some limits on our capacity for processing information. Psychological Review, 63(2), 81-97. doi:10.1037/h0043158

Møller, A. R. (2003). Sensory systems: Anatomy and physiology: Current opinion in neurobiology (Vol. 10). Houston: Gulf Professional Publishing.

Nakamura, A., Yamada, T., Goto, A., Kato, T., Ito, K., Abe, Y., ..., Kakigi, R. (1998). Somatosensory homunculus as drawn by MEG. NeuroImage, 7(4), 377-386. doi:https://doi.org/10.1006/nimg. 1998.0332

Narici, L., Modena, I., Opsomer, R. J., Pizzella, V., Romani, G. L., Torrioli, G., ... Rossini, P. M. (1991). Neuromagnetic somatosensory homunculus: A non-invasive approach in humans. Neuroscience Letters, 121(1/2), 51-54. doi:https://doi.org/10.1016/0304-3940(91) 90647-C

Olivers, C. N. L., \& Watson, D. G. (2008). Subitizing requires attention. Visual Cognition, 16(4), 439-462. doi:https://doi.org/10.1080/ 13506280701825861

Overvliet, K. E., \& Plaisier, M. A. (2016). Perceptual grouping affects haptic enumeration over the fingers. Perception, 45(1/2), 71-82. doi:https://doi.org/10.1177/0301006615594958

Pagano, S., Lombardi, L., \& Mazza, V. (2014). Brain dynamics of attention and working memory engagement in subitizing. Brain Research, 1543, 244-252. doi:https://doi.org/10.1016/j.brainres. 2013.11.025

Piazza, M., Fumarola, A., Chinello, A., \& Melcher, D. (2011). Subitizing reflects visuo-spatial object individuation capacity. Cognition, 121(1), 147-153. doi:https://doi.org/10.1016/j.cognition.2011.05.007

Piazza, M., Mechelli, A., Butterworth, B., \& Price, C. J. (2002). Are subitizing and counting implemented as separate or functionally overlapping processes? NeuroImage, 15(2), 435-446. doi:https:// doi.org/10.1006/nimg.2001.0980

Plaisier, M. A., \& Smeets, J. B. J. (2011). Haptic subitizing across the fingers. Attention, Perception \& Psychophysics, 73(5), 1579-1585. doi:https://doi.org/10.3758/s13414-011-0124-8

Plaisier, M. A., Tiest, W. M. B., \& Kappers, A. M. L. (2009). One, two, three, many-Subitizing in active touch. Acta Psychologica, 131(2), 163-170. doi:https://doi.org/10.1016/j.actpsy.2009.04.003

Plaisier, M. A., Tiest, W. M. B., \& Kappers, A. M. L. (2010). Grabbing subitizing with both hands: Bimanual number processing. Experimental Brain Research, 202(2), 507-512. doi:https://doi. org/10.1007/s00221-009-2146-1

Poiese, P., Spalek, T. M., \& Di Lollo, V. (2008). Attentional involvement in subitizing: Questioning the preattentive hypothesis. Visual
Cognition, 16(4), 474-485. doi:https://doi.org/10.1080/ 13506280801969676

Railo, H., Koivisto, M., Revonsuo, A., \& Hannula, M. M. (2008). The role of attention in subitizing. Cognition, 107, 82-104. doi:10.1016/ j.cognition.2007.08.004

Repp, B. H. (2007). Perceiving the numerosity of rapidly occurring auditory events in metrical and nonmetrical contexts. Perception \& Psychophysics, 69(4), 529-543. doi:https://doi.org/10.3758/ BF03193910

Riggs, K. J., Ferrand, L., Lancelin, D., Fryziel, L., Dumur, G., \& Simpson, A. (2006). Subitizing in tactile perception. Psychological Science, 17(4), 271-272. doi:https://doi.org/10.1111/j.1467-9280. 2006.01696.x

Rinaldi, L., Di Luca, S., Henik, A., \& Girelli, L. (2016). A helping hand putting in order: Visuomotor routines organize numerical and nonnumerical sequences in space. Cognition, 152, 40-52. doi:https:// doi.org/10.1016/j.cognition.2016.03.003

Saltzman, I. J., \& Garner, W. R. (1948). Reaction time as a measure of span of attention. Journal of Psychology: Interdisciplinary and Applied, 25(2), 227-241. doi:https://doi.org/10.1080/00223980. 1948.9917373

Sengupta, R., Bapiraju, S., \& Melcher, D. (2016). Big and small numbers: Empirical support for a single, flexible mechanism for numerosity perception. Attention, Perception, \& Psychophysics, 79(1), 253266. doi:https://doi.org/10.3758/s13414-016-1221-5

ten Hoopen, G., \& Vos, J. (1979). Effect on numerosity judgment of grouping of tones by auditory channels. Perception \& Psychophysics, 26(5), 374-380. doi:https://doi.org/10.3758/ BF03204162

Trick, L. M., \& Pylyshyn, Z. W. (1994). Why are small and large numbers enumerated differently? A limited-capacity preattentive stage in vision. Psychological Review, 101(1), 80-102. doi:https://doi.org/10. 1037/0033-295X.101.1.80

von Glasersfeld, E. (1982). Subitizing: The role of figural patterns in the development of numerical concepts. Archives de Psychologie, 50, 191-218. Retrieved from http://psycnet.apa.org/record/1983$12449-001$

Wilson, M. (2002). Six views of embodied cognition. Psychonomic Bulletin \& Review. Retrieved from http://www.springerlink.com/ index/GT33335RG5156860.pdf

Wolters, G., van Kempen, H., \& Wijlhuizen, G.-J. (1987). Quantification of small numbers of dots: Subitizing or pattern recognition? The American Journal of Psychology, 100(2), 225-237. doi:https://doi. org/10.2307/1422405

Yang, T. T., Gallen, C. C., Schwartz, B. J., \& Bloom, F. E. (1993). Noninvasive somatosensory homunculus mapping in humans by using a large-array biomagnetometer. Proceedings of the National Academy of Sciences, 90(7), 3098-3102. doi:https://doi.org/10. 1073/pnas.90.7.3098 